\title{
IL 'GIOCO’ INTERCULTURALE TRA LINGUA E LINGUAGGIO NE LA LUPA DI VERGA E IN BODAS DE SANGRE DI LORCA
}

DOI: http://dx.doi.org/10.12775/TSP-W.2020.011

Date of receipt: 23.05 .2020

Date of acceptance: 31.10 .2020

The intercultural 'game' between language and language in La Lupa by Verga and in Bodas de sangre by Lorca - This article aims to compare and analyze the language issues and solutions as well as the way the presented world is constructed through the usage of language within a Spanish literary trend, Generación del 27, and Italian Realism. The analyzed works are theatrical plays Bodas de sangre by Federico García Lorca and La Lupa by Giovanni Verga. The conducted analysis focuses on the usage of regional languages and dialects, and their transposition (or lack thereof) into a national language in the fields of semantics, semiotics, and stylistics. The case study also includes elements of the authors' biographies and takes into consideration their interest in sociology of the unprivileged class and ethnology. The article introduces and compares the evolution of the theatrical play in southern Italy and Spain and emphasizes the similarities and concurrences which have not been given sufficient attention in literary studies.

Keywords: Giovanni Verga, La Lupa, Federico Garcia Lorca, Bodas de sangre, folclore, village, theater, Italian theater, Spanish theater, 19th century, 20th century.

Międzykulturowa ,gra” między językiem a językiem w La Lupa Vergi oraz w Bodas de Sangre Lorci. Niniejszy artykuł ma na celu porównanie i analizę proble-

${ }^{1}$ Dominika Machel - Department of Italian Literature and Literatures in Spanish, Faculty of Humanities, Nicolaus Copernicus University in Toruń. 
mów i rozwiązań językowych oraz sposobu konstruowania świata przedstawionego za pomocą języka w ramach hiszpańskiego nurtu literackiego, Generación del 27 i włoskiego realizmu. Analizie poddane są sztuki teatralne Bodas de sangre Federico García Lorci i La Lupa Giovanniego Vergi. Przeprowadzona analiza skupia się na wykorzystaniu języków regionalnych i dialektów oraz ich transpozycji (lub jej braku) na język narodowy w zakresie semantyki, semiotyki i stylistyki. Studium przypadku zawiera również elementy biografii autorów i uwzględnia ich zainteresowania socjologią klasy nieuprzywilejowanej i etnologią. W artykule przedstawiono i porównano ewolucję sztuki teatralnej w południowych Włoszech i Hiszpanii oraz podkreślono podobieństwa i zbieżności, którym nie poświęcono do tej pory wystarczającej uwagi w badaniach literackich.

Słowa kluczowe: Giovanni Verga, La Lupa, Federico Garcia Lorca, Bodas de sangre, folklor, wieś, teatr, teatr włoski, teatr hiszpański, wiek XIX, wiek XX.

In questo saggio tratteremo la visione del mondo rurale, nonché le sfumature della lingua, delle intrusioni dialettali e del linguaggio colloquiale contadino rappresentati in due opere teatrali: La Lupa del drammaturgo siciliano Giovanni Verga e Bodas de sangre del tragediografo andaluso Federico García Lorca, esaminando anche gli aspetti macro e microstrutturali dei testi.

L'Italia e la Spagna sono paesi vicini a livello geografico e socio-culturale geograficamente e socio-culturalmente, legati alla storia del Mar Mediterraneo e dalla comune matrice linguistica latina. María de las Nieves Muñiz Muñiz riporta che "all'Italia e alla Spagna, parimenti arretrate e solari, anche se diversamente 'antiche', toccò il ruolo di serbatoio di miti, leggende e cultura popolare del Mediterraneo"2. Partendo da tale presupposto ci concentreremo su uno studio comparato di carattere interculturale riguardante il teatro italiano e spagnolo a cavallo tra Otto e Novecento. Prima di tutto va sottolineato che, prima dell'apparizione del verismo in Italia $^{3}$, l'area rurale e i suoi abitanti erano, fin dall'antichità, marginalizzati e non degni d'interesse letterario. Così, su questo fenomeno, esprime la propria opinione Leonardo Sciascia:

La letteratura siciliana ha avuto sempre come materia e come oggetto la Sicilia. Una Sicilia che non e comunque un eden della memoria, ma il luogo di concrete pratiche sociali [...]; sulla Sicilia non si è mai proiettata la luce dell'utopia, ma l'amarezza della denuncia, appena mitigata dalle magie tonali della favola e della

${ }^{2}$ Muñiz Muñiz, María de las Nieves, 1999, Italia e Italie. Immagini tra Rivoluzione e Restaurazione, Roma, Bulzoni, p. 162.

3 Zapullo Muscará, Sarah, Invito alla lettura di Verga, Milano, Mursia, 1997, p. 92. 
reverie. Ogni vicenda storica e stata osservata dallo scrittore siciliano alla luce della negazione ${ }^{4}$.

Anche in Spagna dominava la "visión aún predominante [que] niega protagonismo alguno al mundo rural y al campesinado en la construcción nacional, en la formación cultural o en la modernización socioeconómica de nuestro país" ${ }^{5}$. La plebe era considerata un genere umano più basso, quasi non umano ma animale (a tal proposito, non si dimentichi che il titolo dell'opera verghiana è, allo stesso tempo, sia il nome di un animale che il soprannome della protagonista). Era comune l'idea che il ceto contadino fosse "persuadido de que nadie remediará sus infortunios y de que sus únicos derechos son obedecer, pagar, sufrir y callar"6.

È particolarmente interessante, in questo caso, condurre una ricerca fatta sulle opere teatrali di due importanti scrittori come Verga e García Lorca, entrambi originari di regioni poste all'estremo sud dei loro rispettivi paesi, legati al folclore e alla cultura contadina e promotori delle classi subalterne, nonché denunciatori dell'ingiustizia governante "il mondo dei vinti" ", per l'appunto il mondo rurale.

Tanto La Lupa quanto Bodas de sangre (Nozze di sangue) sono ambientati nella realtà di un arretrato paese del sud in cui regnano pregiudizi, superstizioni, sfruttamento, tradizioni e rigide norme sociali. Gli autori non idealizzano il mondo rurale, ma denunciano la miseria della vita contadina e descrivono le emozioni alte e basse della plebe fino ad allora trascurate dalla letteratura.

Si cerca dunque di mettere in luce il percorso fatto dagli autori nell' adoperare un linguaggio e uno stile che permetta di rappresentare il mondo contadino in un modo più verosimile e comprensibile al pubblico.

${ }^{4}$ Zappulla, Angelo, La Giustizia nella Letteratura e nello Spettacolo Siciliani tra '800 e '900. Da Verga a Sciascia, Catania, Istituto di Storia dello Spettacolo Siciliano, 1997, p. 232.

${ }^{5}$ González de Molina, Manuel. Algunas reflexiones sobre el mundo rural y los movimientos campesinos in Historia contemporánea española in Rivera, Antonio, Ortiz de Orruño, José María, Ugarte, Javier. Movimientos sociales en la España Contemporánea. Madrid, Abada Editores, 2008, pag. 97-126. [visione ancora predominante [che] nega qualsiasi ruolo al mondo rurale e ai contadini nella costruzione nazionale, nella formazione culturale o nella modernizzazione socio-economica del nostro paese].

${ }^{6}$ Senado Gomez, Julio. Castilla en escombros: las leyes, las tierras, el trigo y el hambre. Valladolid: Ámbito, 1993, p. 23. [convinta che nessuno rimedierà alle loro sventure e che i loro unici diritti sono obbedire, pagare, soffrire e tacere], traduzione nostra.

7 Cocchiara Giuseppe, La lezione di Pitré, in Storia del folklore europeo, Torino, Boringhieri, 1979, p. 65. 
Lo stesso Verga aveva dichiarato in una lettera all'amico Capuana: "Io te lo ripeterò [le vicende popolari] così come l'ho raccolto pei viottoli dei campi (...), colle medesime parole semplici e pittoresche della narrazione popolare"8. Ciò non era così facile data la situazione linguistica italiana nell'Ottocento - alto tasso di analafabetismo, profonda dialettizzazione e una scarsa conoscenza della lingua standard. Per questo motivo prendiamo in esame i saggi sul dialetto siciliano e sul teatro dialettale.

Nonostante in Spagna non ci fossero problemi con la lingua standard, anche Lorca fa uso delle forme dialettali andaluse e delle influenze zingare nel linguaggio della plebe andalusa. Anche quest'aspetto verrà esaminato in questo articolo.

I due drammaturghi erano avvantaggiati nel descrivere il mondo rurale. Come afferma Pitré': "la conoscenza della lingua locale e popolare è essenziale per comprendere la vita e la visione del mondo degli umili" ${ }^{\prime 10}$. Pitré è stato fonte di ispirazione per due dei più noti scrittori siciliani: Luigi Capuana, che nelle opere di Pitré trovò idee e materiale per le proprie fiabe, e Giovanni Verga che a lui si ispirò per le "tinte schiette"; e proprio grazie al repertorio di Pitré sono nate le particolari usanze del suo mondo dei vinti quali mescolanza degli universi paremiologici specifici per l'italiano e il siciliano e persino le trame di alcune novelle come Guerra di Santi.

Ai fini di questo studio, occorre esaminare la seguente accezione della definizione fornita da Treccani in merito ad entrambi i concetti di linguaggio e lingua che, in un certo senso, si sovrappongono:

[Linguaggio] Modo individuale di esprimersi, sia per un uso particolare della lingua, considerato sotto l'aspetto formale (1. raffinato, volgare, triviale, affettivo, dialettico, ecc.), sia per l'uso di un proprio codice linguistico (1. convenzionale; 1. infantile; talora con riguardo a caratteri stilistici: il 1. di Dante, di Ariosto, di D’Annunzio, ecc.). Anche di una classe d'individui, di un determinato ambiente professionale o gruppo sociale (con riguardo al lessico, alla fraseologia, alla nomenclatura, ecc.):

${ }^{8}$ Lettera n. 108, Milano, 25 febbraio 1881, in Raya, Gino, Carteggio Verga-Capuana, Roma, Edizioni dell'Ateneo, 1984, p. 108.

${ }^{9}$ Giuseppe Pitrè (22 dicembre 1841 a Palermo - 10 aprile 1916 a Palermo) era uno scrittore, medico, scrittore, etnologo e senatore del Regno d'Italia. È noto per il suo lavoro pionieristico nei settori del folklore siciliano, della museologia e della cultura materiale, è il più importante ricercatore e studioso delle tradizioni popolari siciliane e l'iniziatore della ricerca folcloristica italiana. Giuseppe Cocchiara, etnologo nonché rettore del Dipartimento di Letteratura e Filosofia di Palermo, e un continuatore del suo lavoro, cosí si esprime del maestro: "la sua opera monumentale resta pietra miliare per la ricchezza e la vastità d'informazioni nel campo del folclore, in cui nessuno ha raccolto "come e quanto" lo scrittore palermitano".

${ }^{10}$ Pitré, Giuseppe. Usi e costumi del popolo siciliano, Firenze, Universale Cappelli, 1961, p. 5. 
1. poetico, letterario, filosofico, tecnico, scientifico, militare, marinaresco, burocratico; 1. giornalistico, pubblicitario; il 1. dei medici, degli avvocati; i 1. settoriali, speciali o specialistici; il 1. comune, quotidiano, familiare, popolare, ecc. Spesso s'intende piuttosto il tono, abituale o occasionale, del discorso: nobiltà, semplicità di 1.; tenere o usare un 1. dignitoso, franco, severo, insultante, sconveniente; gli parlò con 1. paterno; che razza di 1. è questo?; ti consiglio di cambiare linguaggio. E in senso fig., con riferimento ad alcune qualità del discorso o al sentimento ispiratore: parlare il 1. del cuore, dei sentimenti, della ragione ${ }^{11}$.

[Lingua] Con accezione più ristretta, il particolare aspetto con cui si configura la lingua in un determinato ambiente, nello stile di uno scrittore, nei successivi stadî della sua evoluzione, o nelle varie sue possibili realizzazioni: l. letteraria, poetica, quella propria dei letterati e dei poeti (anche, linguaggio letterario, poetico; e linguaggio burocratico, giuridico, commerciale, popolare, plebeo, ecc., più spesso che lingua burocratica, ecc.); l. parlata, o dell'uso parlato, quella realizzata oralmente, e più in generale la lingua della comunicazione quotidiana, contrapp. sia alla l. Scritta (quella cioè realizzata con simboli grafici, e in senso più ampio la lingua, più controllata, delle comunicazioni scritte) sia ai varî linguaggi settoriali; l. Viva o dell'uso odierno, quella in uso ancor oggi; l. trasmessa, quella tipica della comunicazione a distanza, nel parlato (telefono, radio, televisione, cinema ecc.) e nello scritto (Internet, posta elettronica ecc.); la l. del Duecento, del Trecento, ecc.; la l. di Dante, di Manzoni ${ }^{12}$.

Siamo quindi interessati alla lingua e al linguaggio in un'ottica d'uso settoriale (lingua o linguaggio degli umili), nonché come varianti linguistiche e stilistiche cosí come vengono usate nelle opere di Lorca e Verga.

\section{SFUMATURE DELLA LINGUA E DEL LINGUAGGIO ADOPERATE DA VERGA NE LA LUPA}

Il problema incontrato da Verga nello scrivere La Lupa fu la mancanza nella tradizione narrativa di un orientamento stilistico grammaticale adatto a descrivere le classi subalterne. Non c'era nessun modello o punto di riferimento da seguire per effettuare uno studio realistico sulla vita contadina. Il problema era

${ }^{11}$ Cfr. Voce "linguaggio" in Vocabolario Treccani http://www.treccani.it/vocabolario/linguaggio/.

12 Cfr. Voce "lingua" in Vocabolario Treccani http://www.treccani.it/vocabolario/lingua/. 
comune e fu affrontato in modo diverso da vari autori. Domenico Comparetti ad esempio affermava di aver riferito "fedelmente come furono narrate" le proprie Novelline popolari italiane (875), dichiarando però di averle "tradotte nella lingua comune per garantirne la circolazione"13. Nel caso dello scrittore siciliano, invece, la studiosa verghiana Gabriella Alfieri che commenta le scelte linguistiche e stilistiche come segue:

La ricerca verghiana di un parlato 'inerente' ai soggetti é una costante irrinunciabile, dall'esordio romantico catanese a romanzi e commedie 'sociali' fiorentini, a novelle e drammi rusticani o suburbani del 'magico' decennio milanese, fino alla produzione postverista. Anche da semplice portavoce del 'press'a poco della narrazione popolare, Verga si distaccava però dalla demologia coeva che sdialettizzava gli etnotesti per 'nazionalizzarli' 14 .

Il drammaturgo catanese poteva solo appoggiarsi ai suoi testi precedenti, oppure consultarsi (cosa che faceva con gran vivacità) con il suo amico e demologo di grande fama Giuseppe Pitrè:

Pitré ci fa conoscere che le fonti alle quali egli [Verga] ha attinto per le sacre rappresentazioni sono la tradizione, i libri e i manoscritti, mentre per le feste s'è servito, ancora una volta, delle tradizioni orali «in tutta l'accezione del vocabolo: leggende, fole, canti, proverbi, usi, credenze, superstizioni; elementi tutti, per chi li consideri, della vita popolare e manifestazioni di essa ${ }^{15}$.

Altro problema per i veristi è costituito dal fatto che la lingua standard adoperata dopo l'unità d'Italia non era ancora totalmente formata né usata in modo omogeneo nelle diverse regioni del Paese. Il riflesso di questa situazione sono taluni dubbi linguistici che si possono osservare, ad esempio, nelle riflessioni dello scrittore napoletano, amico di Verga, Edoardo Scarfoglio:

Tranne i Toscani, tutti gli italiani quando si trovano a discorrere con persone che non siano del loro paese, traducono dal proprio dialetto, e il più delle volte tradu-

${ }^{13}$ Nencioni, G. Antropologia poetica? In Fra grammatica e retorica. Da Dante a Pirandello, Torino, Einaudi, 1983, p. 162.

${ }^{14}$ Alfieri, Gabriella, Dialetto e dialetti in Verga: funzionalizzazione diamesica della diatopia fra narrativa e teatro in Dialetto. Uno nessuno centomila a cura di Gianna Marcato, Padova, CLEUP, 2017, p. 307.

${ }^{15}$ Giancristofaro, Lia. Il segno dei Vinti. Lettura antropologica dell'opera di Verga, https://www. academia.edu/33137043/Il_segno_dei_Vinti__Lettura_antropologica_dellopera_di_Verga, p. 13. 
cono male. Ho notato ultimamente questo fatto nella propria persona di Giovanni Verga. Noi parlammo un giorno lungamente insieme, e io notavo lo stento e l'imperfezione del suo italiano, com'egli, certamente, si scandalizzava della sconcezza del mio. Poi andammo a mangiare delle sarelle sopra una tartana messinese nel porto di Ripa Grande; e subito il Verga cominciò a parlar siciliano coi marinari con una così facile speditezza che io dissi in me medesimo: Diavolo! E perché costui non fa parlar siciliano i Siciliani delle sue opere? ${ }^{16}$

L'autore difatti non voleva far parlare siciliano i suoi personaggi, dato che i dialoghi sarebbero risultati comprensibili solo per un limitato circolo di intellettuali. Rifiutò anche "la pura e semplice trascrizione del dialetto", optando invece per una mediazione tra il linguaggio colto letterario toscano e quello folcloristico e rurale della plebe; così ne La Lupa incontriamo diversi frammenti che non sono apertamente dialettali, ma dialettizzanti e idiomatici ${ }^{17}$.

Grazie al lavoro svolto da Verga per adattare il dialetto alla scrittura e mescolarlo in modo efficace con l'italiano, i dialetti sono entrati nel mondo letterario: "l'autorizzazione letteraria dell'uso del dialetto, o, a dir meglio, il suo riscatto dalle forme e dai generi "subalterni" in cui esso era stato tradizionalmente relegato è, si sa, merito del Verga" ${ }^{18}$. Comunque nonostante gli sforzi, le invenzioni dei pionieri in qualsiasi campo non sono mai perfette. Ciò vale anche per lo scrittore catanese.

Così Giacomo Debenedetti ha commentato le imperfezioni del linguaggio verghiano ne La Lupa:

Nonostante la tessitura spesso abbondante, contaminata da ricercatezze letterarie o dall'uso di metafore estranee all'immaginativa dell'autore ("attacar briga"), i dialoghi conservano però, in alcune proposizioni il carattere asintattico e il vocabolario pregnante del linguaggio locale verghiano: (Atto I quadro 1) Domani si vuol sudare il pane!; per tirarlo in peccato mortale; con due occhi in fronte che erano due stelle!; Benedetta la gnà Pina che porta l'allegria dove va lei; (Atto I, 3) Non vuol sentirci da quell'orecchio!; Ora che avete scaldato le orecchie? Ora che non mi tengo più; (I 5) Ma il cuore, l'avete peggio, anche! ${ }^{19}$

${ }^{16}$ Scarfoglio, Edoardo. Il Libro Di Don Chisciotte - Primary Source Edition, Charleston, Nabu Press, 2014, p. 95.

17 Scarfoglio, Edoardo, op.cit., p. 94.

${ }^{18}$ Cfr. Inproposito, Sansone, Marcello. Relazioni fra la letteratura italiana e le letterature dialettali, in Problemi e orientamenti critici di Lingua e di Letteratura italiana, IV (Letterature comparate), a c. di Momigliano, Arnoldo, Milano, Marzorati Editore, 1947.

${ }_{19}$ Debenedetti, Giacomo. Verga e il naturalismo, Milano, Aldo Garzanti Editore, 1978, p. 28. 
Secondo Pitré, la conoscenza della lingua locale e popolare di Verga è un fattore chiave nel suo atto di creare e raccontare il mondo degli umili, secondo i nuovi termini dell'antropologia moderna ${ }^{20}$. Inoltre, il demologo palermitano conferma la traduzione fedele delle parole siciliane in lingua italiana o, meglio, la trasposizione di esse operata da Verga ${ }^{21}$.

Tra le righe del dramma compaiono spesso le canzoni popolari. Tra queste, i frammenti dei canti popolari, cantati da Bruno e Grazia e Garofano pomposo, si basano sul canto tradizionale siciliano Garofulu puтрusu o Jarufalu puтрusu ${ }^{22}$ che cantava ripetutamente la Lupa:

Garofano pomposo, dolce amore, dimmelo tu come ti debbo amare!

Tu di nascosto m'hai rubato il cuore ed io qui venni se mel vuoi ridare.

E n'ho toccati tanti cuori duri! Solo il tuo non si lascia intenerire!

Ora men vado a governo d'amore... Il mio lo lascio a te. Non ti scordare ${ }^{23}$.

Le canzoni canticchiate in crocchio dai contadini sono un esempio del modo con cui i membri delle società rurali esprimevano le loro emozioni, i dolori, i desideri. Non essendo capaci di esprimersi con parole belle e raffinate, facevano uso della saggezza e della bellezza popolare nascosta tra le rime dei canti folcloristici ${ }^{24}$.

Il canto di "Garofano pomposo" è l'unica dichiarazione esplicita dell'amore della Lupa verso il genero. L'altro mezzo linguistico con cui Verga dipinge la sua visione del mondo contadino nell'opera teatrale in oggetto è il proverbio. Quasi ogni pagina è adornata da proverbi e detti siciliani dando un particolare colorito sia ai personaggi che alle vicende:

La poesia de La Lupa si nutre dei proverbi che nascono dall'esperienza tramandata e rivissuta. Le idee, in un mondo che coltiva tradizioni familiari arcaiche, hanno un immediato riferimento alle cose e sono saggezza antica, somma di esperienze, un modo di farsi capire e di cantare, con tristezza o allegria, la vita. L'uomo è in ciò che fa, negli impegni, nella autorità delle tradizioni, nella realtà storica del suo stato che condiziona sentimenti e pensieri ${ }^{25}$

${ }^{20}$ Pitrè, Giuseppe.Usi e costumi, credenze e pregiudizi del popolo siciliano, Firenze, Barbera, 1944-1952, pp. 23-24.

${ }^{21}$ Giancristofaro, Lia, op.cit., p. 13.

${ }_{22}$ Pitrè, Giuseppe., Canti popolari siciliani, Bologna, Forni, 1980, p. 78.

${ }^{23}$ Verga, Giovanni, La lupa in Tutto il teatro, Milano, Arnoldo Mondadori Editore, 1980, op.cit., p. 21.

${ }^{24}$ Ibidem.

25 Pitrè, Giuseppe. op.cit., p. 89. 
Qui di seguito riportiamo alcuni esempi di proverbi citati nelle battute dei contadini di modo da rappresentare una sintesi della saggezza rurale adoperata nell'universo verghiano: (Atto I scena 2) Vile che si pente, compare Janu!; il buon panno fino allla cimosa!; (Atto I scena 7) Maledetto chi si pente!; Meglio cavarselo subito il dente che duole! Gli altri proverbi comparsi nel dramma sono citati invece esplicitamente tali da chi li usa (Atto I scena 1)"Ai miei tempi si diceva..."; sapete come dice il proverbio?"), fra virgolette (Atto I scena 1) “L'uomo è il fuoco, la donna la stoppa, viene il diavolo e soffia!") oppure introdotti tramite uso del corsivo (Atto I scena 3) "Chi non mi vuole non mi merita"; "Chi tarde arriva, amle alloggia", (Atto I scene 7) "dalla spina nasce la rosa", Comare Pina, sapete come dice il proverbio: "Maritati e muli lasciali soli" 26 .

Resta ovvio che nell'opera vengano usate tutte le parti del discorso che possono riguardare la vita rurale. A volte le frasi costituiscono una fotografia della vita contadina come: "Tu, pensa alle bestie! Vedi se hanno mangiato l'orzo, e da' la paglia fresca prima di andare a dormire. Hai inteso?". Diamo uno sguardo ad alcuni altri esempi tra cui: verbi - mietere, zappare, governare le bestie, uggiolare dei cani, il pronome - essa che "vi è usato come pronome personale, secondo un uso che si trova nei dialetti meridionali, in quello napoletano soprattutto (issa), resistendo nel siciliano un idda che facilmente si intuisce affine all' italiano "ella". Verga sa però che in siciliano idda è generico per persona, per animale e per cosa, dove ella è in italiano solo pronome personale. Di qui l'assimilazione di idda a essa." ${ }^{27}$, inoltre c'è una "vasta gamma del lessico che si riferisce al mondo del lavoro e della vita contadina" 28 articolata in aggettivi e nomi: pagliericcio, ballo tondo, aia, capanna, biade mature, grembiule, attrezzi rurali.

Analizziamo lo studio sul lessico de La Lupa svolto da Ludovico Fulci ${ }^{29}$. Alla vita dei campi fanno riferimento anche i termini relativi agli oggetti della vita quotidiana in un paese siciliano. La Lupa conosce Nanni lavorando con lui "nelle chiuse del notaro" cioè nelle terre di proprietà di un notaio. Nanni "spinge con la pala le olive sotto la macina", l'aia (il terreno spianato davanti alle case dei coloni) è lo scenario complice degli amori di Nanni e della gnà Pina. Se dagli esterni passiamo agli interni, troviamo chiari riferimenti alla vita nei campi. Dando la figlia in sposa a Nanni, la Lupa dichiara che per lei basta un "cantuccio" (angolo) dove stendere un "pagliericcio" (un po' di paglia su cui sdraiarsi

${ }^{26}$ Barsotti, Anna, Verga drammaturgo. Tra commedia borghese e teatro verista siciliano, Firenze, La Nuova Italia Editrice, 1974, p. 134-135.

${ }^{27}$ Debenedetti, Giacomo. op.cit., p. 279.

${ }^{28}$ Fulci, Ludovico, La Lupa di Verga. Esercitazioni di lettura e spunti di approfondimento, Wrocław, Dolnośląskie Wydawnictwo Edukacyjne, 2005, pp. 15-16.

${ }^{29}$ Ibidem. 
per dormire). Ancora: gli occhi della Lupa sono neri come il carbone, e sia la paglia che il carbone sono tipici prodotti di una comunità contadina. La paglia si raccoglie e serve per intrecciare canestri, fabbricare scope, sedie, piccolo mobilio; il carbone si ottiene facendo bruciare la legna in appositi forni costruiti vicino ai boschi e si utilizzerà in inverno come combustibile. Rientrano tra gli oggetti della vita quotidiana, trascorsa negli spazi della casa, l'olmo e la scure che vi è attaccata. Altre espressioni fanno riferimento alla situazione climatica e atmosferica: "Le ore caldo di giugno, fosse stato greco e levante di gennaio, oppure scirocco di agosto". ${ }^{30}$

Abbiamo citato solo alcuni esempi che illustrano la ricchezza delle pagine verghiane per accennare a come "il linguaggio verghiano si articola in un disteso racconto della natura, del paese siciliano della vita, con suoni e sensazioni sovrapposte; conserverà sempre la concretezza del fare, dell'esistere - ritratto, scena, azione - di un'ottica che coglie le cose e gli uomini nel loro divenire naturale e sociale" $"$.

\section{SFUMATURE DELLA LINGUA E DEL LINGUAGGIO ADOPERATE DA GARCÍA LORCA IN BODAS DE SANGRE}

Lorca aveva dubbi sul linguaggio da usare per scrivere del mondo rurale. Il principale tra questi dubbi era decidere se dedicare la sua opera ad un pubblico vasto o meno.

Il linguaggio poetico è caratterizzato da deviazioni e straniamento rispetto alla lingua standard; così Lázaro Carreter ${ }^{32}$ afferma che il problema diventa ancor più complicato quando entra in gioco la poesia rurale e la redazione di questa. In questi aspetti Lorca si allontana dall'uso tradizionale o regolare del linguaggio. La definizione di questo suo uso del linguaggio è il punto principale di molte discussioni. In effetti, molti studiosi sostengono che lui costituisce una lingua tutta sua: "aunque no sea posible definir en términos metódicos el lenguaje literario, a través del estudio de la poesía de autores específicos, resultará posible alcanzar convicciones científicamente valiosas acerca de las diferencias entre el idioma de los escritores y el estándar" ${ }^{33}$.

\footnotetext{
${ }^{30}$ Fulci, Ludovico, op.cit., p. 15.

${ }^{31}$ Ferrante, Luigi, Verga, Milano, Nuova accademia, 1964, p 70.

${ }^{32}$ Lázaro Carreter, Fernando, Correa Calderón, Evaristo, Cómo se comenta un texto literario, Madrid, Ediciones Cátedra, 1975, p. 197.

33 Travalia, Caroline, Pazo, José, Juego, léxico y sintaxis en la lengua de García Lorca, p. 27. https://www.academia.edu/12677581/Juego_1\%C3\%A9xico_y_sintaxis_en_la_lengua_de_
} 
Lorca era un individuo forte ed indipendente che elaborò un linguaggio proprio "compenetrando el corazón español con el alma gitanesca ha trazado las posibilidades de su tierra del lado del dramatismo y la agitada movilidad. Es, pues, su poesía a la vez la labor efinada del orfebre y el desgarrado canto jondo [miscelando il cuore spagnolo con l'anima zingara, ha disegnato le possibilità della sua terra dal lato del dramma e della mobilità frenetica. È, quindi, la sua poesia allo stesso tempo il raffinato lavoro dell'orafo e il canto jondo straziante" 34 . Essendo testimone oculare della misera vita dei contadini andalusi, Lorca provava un elementare senso di ingiustizia derivante dalla contrapposizione tra ricchi e poveri, il che si aggiungeva al "gusto di disegnare motivi popolari" 35 e a una profonda ricezione della natura e delle questioni della vita: amore, passione, miseria, sangue, morte ${ }^{36}$. Non sorprende quindi che il drammaturgo granadino abbia elaborato un linguaggio e uno stile particolare, una sintesi di tutta la ricca tradizione artistica che, come nei grandi talenti creatisi da sé, si trasforma nel suo linguaggio specifico. In questo senso, va sottolineata l'importanza di Lorca come creatore di un nuovo linguaggio poetico, dove parola e immagine, proprio come la fantasia e la realtà, acquisiscono contenuti completamente nuovi ${ }^{37}$.

Nel linguaggio adoperato in Bodas de sangre l'autore mostra una ricercatezza nell'adozione della parola popolare e, allo stesso tempo, propone una descrizione veritiera delle condizioni di vita presenti nelle zone rurali; mostra anche una transizione all'uso dei costumi andaluso, colorato e pittoresco, e alla profonda bellezza della poesia popolare. Il canto della parola e le qualità dello stile di Lorca sono strettamente legati alla sua conoscenza dell' autentica poesia popolare $^{38}$.

Una studiosa polacca, Beata Baczyńska, nota che l'opera di Lorca costituisce un certo stacco, dal resto degli artisti della generación del 27 a esclusione di Alberti e sottolinea che "Lorca scrive poesie di sensazioni, più musicali e legate a temi reali, con un effetto artistico e sociale piuttosto che spirituale" ${ }^{" 39}$.

Garc\%C3\%ADa_Lorca [anche se non è possibile definire in termini letterari la lingua letteraria, attraverso lo studio della poesia di autori specifici, sarà possibile raggiungere convinzioni scientificamente valide in merito alle differenze tra il linguaggio degli scrittori e quello standard]

${ }^{34}$ Baczyńska, Beata. Historia literatury hiszpańskiej. PWN, Warszawa, 2014, p. 435.

${ }^{35}$ Ivi, p. 636.

${ }^{36}$ Cirese, Alberto Mario, Oggetti, segni, musei : sulle tradizioni contadine, Torino, Einaudi, 1977, p. 89.

37 Ibidem.

${ }^{38}$ Cano, José Luis, Garcia Lorca : biografía ilustrada, Barcelona, Ediciones Destino, 1969, p. 304 .

39 Alonso, Dámaso, op.cit., p. 31. 
Il poeta granadino poteva parlare delle campagne non solo grazie alle sue esperienze dirette vissute a Fuentevaqueros, ma anche grazie alle lezioni di musica (Federico fin da bambino prendeva lezioni di piano e, in età adulta, fece concerti riconosciuti a livello nazionale ${ }^{40}$ ) che gli permisero di portare avanti lo studio dei testi musicali folcloristici. Infatti, sempre secondo Baczyńska ${ }^{41}$ Lorca:

[...] aveva un'educazione musicale che gli ha permesso di affrontare seriamente la cultura popolare non solo dell'Andalusia, ma di tutta la Spagna. Ha presentato le sue osservazioni etnografiche durante conferenze pubbliche su, tra gli altri, ninne nanne popolari (nanas) - Canciones de cuna españolas ${ }^{42}$, e canti popolari della città di Granada e dintorni - Cómo canta una ciudad de noviembre a noviembre ${ }^{43}$.

È impossibile restare indifferenti di fronte all'eccezionale musicalità e ritmica di Bodas de sangre. L'autore, essendo un intenditore di musica, ha saputo mescolare uno stile assai colto con la magia della musicalità popolare, cosicché testi poetici per il canto basati sul folclore andaluso sono stati inclusi nel dialogo scritto in prosa ${ }^{44}$. Difatti, tra le righe del dramma, compaiono spesso canzoni popolari, ninne nanne, nonché le canzoni utilizzate tipicamente per il rito del matrimonio.

La ninna nanna piena di significato e simboli cantata due volte da la Mujer e la Suegra ha radici realistiche dato che Lorca era fortemente interessato a questo genere come evidenzia l'autore stesso:

Hace unos años, paseando por las inmediaciones de Granada, oí cantar a una mujer del pueblo mientras dormía a su niño. Siempre había notado la aguda tristeza de las canciones de cuna de nuestro país, pero nunca como entonces sentí esta verdad tan concreta. Al acercarme a la cantora para anotar la canción observé que era una andaluza guapa, alegre sin el menor tic de melancolía; pero una tradición viva obraba en ella y ejecutaba el mandato fielmente, como si escuchara las viejas voces imperiosas que patinaban por su sangre. Desde entonces he procurado recoger canciones de cuna de todos los sitios de España; quise saber de qué modo dormían a sus hijos las mujeres de mi país ${ }^{45}$.

${ }^{40}$ Cano, José Luis, op.cit., p. 309.

${ }^{41}$ Baczyńska, Beata. op.cit., p. 435.

${ }^{42}$ García Lorca, Federico, conferencia dictada el 13 de diciembre de 1928: Las canciones de cuna españolas. En: Obras completas: México, Aguilar, 1991.

${ }^{43}$ Ibidem.

${ }^{44}$ Baczyńska, Beata. op.cit., p. 315.

${ }^{45}$ García Lorca, Federico, Od pierwszych pieśni do stów ostatnich : (wiersze, proza, listy, 
Le donne di Bodas de sangre addormentavano il figlio di Leonardo con parole tristi, versi crudeli che oggi ci sembrano inadeguati ai bambini, in quanto riguardanti il pianto, le ferite, il sangue e la morte. Questa ninna nanna deprimente è un presagio della tragedia e, allo stesso tempo, un riflesso della dura vita contadina: le condizioni in cui le donne allevavano i bambini erano misere, tali da non permettere di prepararli per un futuro migliore, dunque l'unica cosa che potevano fare era familiarizzare i propri figli con la crudeltà del mondo rurale. Non si dimentichi che le parole nella ninna nanna non sono messe a caso; in Lorca il cavallo è il simbolo della maschilità, la Luna della femminilità, i fiori dell'innocenza e l'acqua stagnante della morte:

SUEGRA Nana, niño, nana del caballo grande que no quiso el agua. El agua era negra dentro de las ramas. Cuando llega al puente se detiene y canta. ¿Quién dirá, mi niño, lo que tiene el agua, con su larga cola por su verde sala?

MUJER (Bajo). Duérmete, clavel, que el caballo no quiere beber.

SUEGRA Duérmete, rosal, que el caballo se pone a llorar. Las patas heridas, las crines heladas, dentro de los ojos un puñal de plata. Bajaban al río. ¡Ay, cómo bajaban! La sangre corría más fuerte que el agua.

MUJER Duérmete, clavel, que el caballo no quiere beber.

SUEGRA Duérmete, rosal, que el caballo se pone a llorar.

MUJER No quiso tocar la orilla mojada, su belfo caliente con moscas de plata. A los montes duros sólo relinchaba con el río muerto sobre la garganta. ¡Ay, caballo grande que no quiso el agua! ¡Ay, dolor de nieve, caballo del alba!

SUEGRA ¡No vengas! Detente, cierra la ventana con rama de sueños y sueño de ramas.

MUJER Mi niño se duerme.

SUEGRA Mi niño se calla.

MUJER Caballo, mi niño tiene una almohada.

SUEGRA Su cuna de acero.

MUJER Su colcha de holanda.

wypowiedzi), a c. di Szleyen, Zofia, Kraków, Wydawnictwo Literackie, 1987, p. 320. [Alcuni anni fa, passeggiando per Granada, ho sentito cantare una donna del villaggio mentre il suo bambino dormiva. Avevo sempre notato la tristezza acuta della ninna nanna del nostro paese, ma mai come allora ho sentito questa verità così concreta. Mentre mi avvicinavo alla donna per annotare la canzone, notai che era una bella andalusa, allegra senza il minimo segno di malinconia; ma una tradizione viva operava in lei e eseguiva fedelmente $i$ comandi, come se ascoltasse le vecchie voci imperiose che scorrevano attraverso il suo sangue. Da allora ho cercato di raccogliere ninne nanne in tutti i posti della Spagna; Volevo sapere come le donne del mio paese addormentavano i loro figli], traduzione nostra. 
SUEGRA Nana, niño, nana.

MUJER ;Ay caballo grande que no quiso el agua!

SUEGRA ¡No vengas; no entres! Vete a la montaña. Por los valles grises donde está la jaca.

MUJER (Mirando). Mi niño se duerme.

SUEGRA Mi niño descansa.

MUJER (Bajito). Duérmete, clavel, que el caballo no quiere beber.

SUEGRA (Levantándose y muy bajito). Duérmete, rosal, que el caballo se pone a llorar. ${ }^{46}$

Il drammaturgo spagnolo, nel creare il suo mondo contadino, fa comportare i suoi personaggi come persone vere, le cui azioni sono quelle dei contadini in carne e ossa. Dunque, osserviamo donne chiuse in casa ad allevare bambini e fare lavori manuali ("Suegra de Leonardo con un niño en brazos. Lo mece. La

${ }^{46}$ García Lorca, Federico, Bodas de sangre, Fuenlabrada (Madrid), Ediciones Cátedra, 2000, p. 14. [SUEGRA Nana, bambino, nana del grande cavallo che non voleva l'acqua. L'acqua era nera dentro i rami. Quando raggiunge il ponte si ferma e canta. Chi dirà, figlia mia, cosa ha l'acqua, con la sua lunga coda nella sua stanza verde?

MUJER (bassa). Vai a dormire, garofano, che il cavallo non vuole bere.

SUEGRA Si addormenta, rosaio, che il cavallo inizia a piangere. Le gambe ferite, la criniera ghiacciata, dentro gli occhi un pugnale d'argento. Scesero al fiume. Oh come stavano andando giù! Il sangue scorreva più forte dell' acqua.

MUJER Addormentarsi, garofano, che il cavallo non vuole bere.

SUEGRA Si addormenta, rosaio, che il cavallo inizia a piangere.

MUJER Non voleva toccare la riva bagnata, il suo caldo caldo con mosche d'argento. Le dure montagne gemettero appena con il fiume morto sopra la gola. Oh, grande cavallo che non voleva l'acqua! Oh, dolore della neve, cavallo all'alba!

SUEGRA Non venire! Fermati, chiudi la finestra con il ramo dei sogni e il sogno dei rami.

MUJER Mio figlio si addormenta.

SUEGRA Mio figlio tace.

MUJER Cavallo, mio figlio ha un cuscino.

SUEGRA La sua culla d'acciaio.

MUJER Il tuo copriletto olandese.

SUEGRA Nana, bambina, nana.

MUJER Oh grande cavallo che non voleva l'acqua!

SUEGRA Non venire; non entrare! Vai in montagna. Attraverso le valli grigie dove si trova il jaca.

MUJER (Guardando). Mio figlio si addormenta.

SUEGRA Mio figlio riposa.

MUJER (breve). Vai a dormire, garofano, che il cavallo non vuole bere.

SUEGRA (crescente e molto corto). Dormi, rosaio, che il cavallo inizia a piangere.], traduzione nostra. 
Mujer, en la otra esquina, hace punto de media" ${ }^{47}$ ), invece agli uomini, anche se costretti a sgobbare nei campi ("En mi tiempo, ni esparto ${ }^{48}$ daba esta tierra. Ha sido necesario castigarla y hasta llorarla para que nos dé algo provechoso"49), sono concessi momenti di svago ("Estuve con los medidores del trigo, siempre entretienen [Ero con i mietitori di grano, sempre mi fanno divertire]"50).

Inoltre, si fa riferimento alla miseria economica della plebe rurale quando una ragazza arriva a casa di Leonardo con una notizia, presentata come importante, riguardante il regalo che fatto il Novio a la Novia. Per gli standard di oggi, appare incredibile che i collant siano visti come un regalo lussuoso:

SUEGRA ¿Qué pasa?

MUCHACHA Llegó el NOVIO a la tienda y ha comprado todo lo mejor que había. SUEGRA ¿Vino solo?

MUCHACHA No, con su madre. Seria, alta. (La imita). Pero ¡qué lujo!

SUEGRA Ellos tienen dinero.

MUCHACHA ¡Y compraron unas medias caladas! ¡Ay, qué medias! ¡El sueño de las

mujeres en medias! Mire usted: una golondrina aquí (señala al tobillo), un barco aquí (señala la pantorrilla), y aquí una rosa (señala al muslo) $)^{51}$

Abbiamo citato solo qualche esempio che illustra il linguaggio adoperato da Lorca in Bodas de sangre, comunque conforme alla linea principale delle opere del grande autore granadino "El tema del destino trágico, la imposibildad de realización, sería el elemento que da unidad profunda a su producción teatral" 52 .

${ }^{47}$ [La suocera di Leonardo con un bambino in braccio. Lo culla. La donna, nell'altro angolo, lavora a maglia], traduzione nostra.

48 Tipo di pianta da cui si fanno canestri.

49 García Lorca, Federico, op.cit., p. 43. [Ai miei tempi, ni esparto dava questa terra. È stato necessario punirla e persino piangere perchè ci dia qualcosa di utile], traduzione nostra.

${ }^{50}$ Ivi, p. 26.

${ }^{51}$ Ivi, p. 24. [SUEGRA Che succede?

RAGAZZA La SPOSA è arrivata al negozio e ha comprato tutto il meglio che c'era.

SUEGRA Sei venuto da solo?

RAGAZZA No, con sua madre. Sarebbe alto. (La imita) Ma che lusso!

SUEGRA Hanno soldi.

RAGAZZA E hanno comprato dei calzini! Oh, che calze! Il sogno del donne in calze! Guardati: una rondine qui (indica la caviglia), a

la barca qui (indica il polpaccio) e qui una rosa (indica la coscia, traduzione nostra.

${ }^{52}$ Luis Sanchez, Francisco, Pensamiento en la literatura española, Poznań, Wydawnictwo Naukowe UAM, 1992, p. 193. [Il tema del tragico destino, l'impossibilità della realizzazione, sarebbe l'elemento che dà profonda unità alla sua produzione teatrale.], traduzione nostra. 
La caratteristica più risaltante del linguaggio del dramma in oggetto resta l'arte con cui gli elementi musicali e visivi, reali e irreali, naturali e stilizzati, si mescolano nelle sue opere.

Ci sono varie tendenze e tradizioni poetiche all'interno dell'opera: "folcloristica e andalusa, realistica e romantica, con lo sforzo di fondere una profonda tradizione della poesia popolare basata sui tesori della poesia anonima ancora viva nelle canzoni popolari". ${ }^{53}$

\section{COMPARAZIONI DELLE VARIANTI DELLE LINGUE E DEI LINGUAGGI IN VERGA E LORCA}

Verga, ne La Lupa, ritrae fedelmente lo stile di vita, le emozioni, il linguaggio e le usanze siciliane, mostrando così, alla borghesia e al pubblico italiano post risorgimentale, le condizioni di miseria nelle quali versava la società rurale siciliana e "allo stesso tempo offrendo ai posteri un importante patrimonio cognitivo sugli usi, i costumi e la mentalità del popolo siciliano alla fine dell'Ottocento" ${ }^{4}$. Lorca, invece, in Bodas de sangre, offre un quadro della provincia meridionale spagnola dipingendola come marginalizzata, arretrata e con "contaminazioni" di origine zingara. Era un mondo governato dall'ingiustizia sociale, dal divario economico e dall'attaccamento alle superstizioni; realtà di cui il drammaturgo voleva rendere coscienti le classi privilegiate spagnole attraverso la triste denuncia sociale: "García Lorca ha vissuto con grande forza tutte le tragedie della sua bellissima terra natale, e questa è probabilmente la fonte della nota di profonda tristezza che domina nel suo lavoro" ${ }^{\circ 5}$.

Sono dunque evidenti le affinità tra le motivazioni e gli scopi delle due rappresentazioni del mondo contadino condotte da Verga e Lorca. Entrambi, nonostante provenissero da famiglie agiate, fin dall'infanzia avevano un grande interesse per la campagna che si è poi trasformato in predilezione per l'adattamento dei motivi folcloristici per un vasto pubblico ${ }^{56}$ nella loro produzione letteraria. Così leggiamo del drammaturgo catanese: "Verga, forse a sua insaputa, possiede i requisiti di base indispensabili, secondo quanto diceva Pitré, a chi si voglia occupare di tradizioni popolari, ossia le osservazioni dirette e personali" ${ }^{57}$. Dell'autore granadino invece possiamo constatare che "Le sue molte letture e

${ }^{53}$ Ibidem.

${ }^{54}$ Pitré, Giuseppe, op.cit., p. 17.

${ }^{55}$ Ibidem.

56 Nicoll, Allardyce. Dzieje teatru, Warszawa, Państwowy Instytut Wydawniczy, 1959, p. 59.

${ }^{57}$ Pitré, Giuseppe, op.cit., p. 5. 
i suoi molteplici viaggi in lungo e in largo per la Spagna, modellano chiaramente gli interessi e le preferenze del giovane poeta nei confronti del folclore andaluso e della vita del suo villaggio" 58 .

Anche se questo studio non è una ricerca etnografica vogliamo comunque mettere in risalto le somiglianze, fino ad ora trascurate negli studi letterari, tra il mondo contadino italiano e quello spagnolo nella prospettiva della loro rappresentazione da parte di Verga e Lorca. Degna di nota è l'analogia riguardante un particolare fattore linguistico ovvero i titoli e gli appellativi che gli autori usano per riferirsi ai propri personaggi. Verga usa titoli di provenienza latino-spagnola, residui della presenza spagnola in Sicilia. In questo caso vale la pena citare l'opinione di Dardano e Trifone i quali così definiscono il flusso lessicale tra spagnolo e italiano:

I cento anni che vanno dalla pace di Cateau-Cambrésis (1559) alla pace dei Pirenei (1659) segnano il predominio della Spagna in Italia (Ducato di Milano, Stato dei Presìdi, Regno di Napoli, Sicilia, Sardegna). È questo il periodo che vede entrare in Italia la maggior parte dei vocaboli spagnoli. Ma anche nei secoli precedenti la presenza aragonese (lingua catalana) nell'Italia meridionale e altri fattori avevano assicurato l'ingresso di alcuni iberismi (vocaboli spagnoli, catalani e portoghesi). (...) Molti ispanismi, d'altra parte, penetrarono nel tessuto lessicale dei dialetti del Sud; sono parole che denominano azioni usuali o oggetti della vita quotidiana: solo per fare alcuni esempi: cartera, comare, compare, vorraccio, ammoinare, gnà, carnizzeri, carpetta, puntapedi, carignu. ${ }^{59}$

Nell'opera di Verga compaiono le seguenti parole di provenienza spagnola: compare, comare, gnà. Citiamo le definizioni di Treccani:

compare "Titolo che si dà a un vecchio amico o a chi, anche occasionalmente, si considera come tale: bevi, compare! Nella novellistica popolare, c. orso, c. lupo, ecc. ", comare "Vicina di casa, legata da rapporti di lunga amicizia e confidenza; anche spreg.: ciarle, pettegolezzi da comari. Appellativo che nelle favole si aggiunge talora a nomi di animali : c. volpe. “, gnà ”[adattam. grafico dello spagn. ña, abbrev. di doña, dueña «donna»]. - Appellativo che nei dialetti siciliani e calabresi significa «signora», ed è prefisso personale per donne di condizione popolana (spesso usato da Verga)."

${ }^{58}$ García Lorca, Federico, Bodas de sangre; con cuadros cronológicos, intr., bibliografía, texto íntegro, notas y llamadas de atención, documentos y orientaciones para el estudio a c. di Manuel Cifo González, Madrid, Editorial Castalia, 1999, p. 6.

${ }_{59}$ Dardano Maurizio, Trifone Pietro. op.cit., pp. 642-643. 
Gli appellativi usati da Verga non sono molto lontani dai corrispondenti termini spagnoli in voga nelle zone rurali della Spagna ai tempi di Lorca e utilizzati da quest'ultimo in Bodas de sangre: compare - compadre, comare - comadre, gnà - doña. Un'altra similitudine interessante riguarda gli aspetti socio-musicali del linguaggio del mondo rurale. Come detto, il mondo contadino non offriva ai suoi abitanti nient'altro che non fosse lavoro sodo e fame. Nel poco tempo libero, i contadini si dedicavano a racconti, fiabe e canzoni: forme di divertimento legate alle parole e alla lingua ${ }^{60}$. Questi aspetti si ritrovano anche nelle due opere qui trattate. A volte in Lorca viene canticchiata una ninna nanna che secondo Sirvent del Otero "Era a menudo todo lo que una madre podía ofrecer a su hijo" ${ }^{1}$. Lo stesso Lorca aveva un'idea simile: "No debemos olvidar que la canción de cuna está inventada (y sus textos así lo expresan) por las pobres mujeres cuyos niños son para ellas una carga, una cruz pesada con la cual muchas veces no pueden" ${ }^{2}$. I canti e le canzoni cantate da Grazia, Bruno e Pina ne La Lupa hanno una ritmica e una struttura simili a quelle cantate dai personaggi di Bodas de sangre prima e durante le nozze. La Zia Filomena racconta una fiaba che si trasforma in un ballo tondo, mentre, in Bodas de Sangre, i rimi del rito di passaggio si trasformano in baile de rueda, la versione spagnola del ballo tondo italiano. Anche le tematiche delle produzioni orali sopra menzionate presentano delle somiglianze semantiche:

I testi ripropongono gli stereotipi ricorrenti in questo genere poetico-musicale: predizione di fausti destini ai nuovi nati, richiesta di protezione ai Santi, a Gesù $e$ alla Madonna, richiami in chiave augurale a elementi vegetali (alberi, fiori, erbe), cosmici (sole, luna), alimentari (dolci, carne, pesce) e suntuari (oggetti d'oro e d'argento), minacce di percosse al bimbo che "non vuole dormire" o promesse di doni, invocazione del sonno. ${ }^{63}$

Le azioni degli abitanti della campagna siciliana e andalusa vengono in nati, richiesta di protezione ai Santi, a Gesù e alla Madonna, richiami in chiave augurale

${ }^{60}$ Pitrè, Giuseppe, op.cit., p. 54.

${ }^{61}$ Sirvent del Otero, María Josefa, Actualidd del cuento infantil en España in Petrini, Enzo, Estudio crítico de la literatura juvenil, Madrid, Rialp, 1963, p. 98. [Spesso era tutto ciò che una madre poteva offrire a suo figlio], traduzione nostra.

${ }^{62}$ García Lorca, Federico, Od pierwszych pieśni do słów ostatnich : (wiersze, proza, listy, wypowiedzi), a c. di Szleyen, Zofia, Kraków, Wydawnictwo Literackie, 1987, p. 320. [Non dobbiamo dimenticare che la ninna nanna è inventata (e i suoi testi lo esprimono) dalle donne povere i cui figli sono per loro un peso, una croce pesante con cui spesso non ce la fanno], traduzione nostra.

${ }^{63}$ Bonanzinga, Sergio, La musica di tradizione orale in Lingue e culture in Sicilia a c. di Ruffino, Giovanni, Vol. 2, Palermo, Centro di studi filologici e linguistici siciliani, 2013, p. 194. 
a elementi vegetali (alberi, fiori, erbe), cosmici (sole, luna), alimentari (dolci, carne, pesce) e suntuari (oggetti d'oro e d'argento), minacce di percosse al bimbo che "non vuole dormire" o promesse di doni, invocazione del sonno ${ }^{64}$.

Le azioni degli abitanti della campagna siciliana e andalusa vengono intraprese prendendo in considerazione la saggezza rurale tra cui le superstizioni: fra vespero e nona una sincera donna non può andare in giro, lo sposo deve portare alla sposa azahar (il fiore d'arancio) prima del matrimonio (il fiore d'arancio è il fiore simbolo del patrimonio anche in Italia meridionale) compaiono anche i proverbi "vile chi si pente", "hace las migas a las tres".

L'uso di questo genere di enunciati orali aumenta con lo sviluppo delle due trame, confermando quindi l'applicazione della tecnica basata su "graduales intensificaciones de la letra popular"65 da parte dei tragediografi qui analizzati.

Sia Verga che Lorca, nel redígere le loro opere, hanno affrontato il problema di come trascrivere le realtà contadine nel modo più veritiero e realista possibile. Entrambi sono stati pionieri della lingua letteraria delle plebi del sud dei rispettivi paesi. La soluzione di descrivere il mondo degli umili per il pubblico più colto, scrivendo in un linguaggio parlato, naturale è, come noto, illusoria: i due drammaturghi impiegano il dialogo, non limitandosi alla semplice trascrizione fonematica del parlato dialettale (con forme in siciliano o andaluso), ma "inventano un linguaggio che ha risonanze, misure sceniche e forme ritmiche secondo il tempo del respiro, cosicché, come dice Shakespeare, la "lingua danza sulla lingua" ${ }^{66}$.

\section{BIBIOGRAPHY:}

Baczyńska, Beata. Historia literatury hiszpańskiej. PWN, Warszawa, 2014.

Barsotti, Anna, Verga drammaturgo. Tra commedia borghese e teatro verista siciliano, Firenze, La Nuova Italia Editrice, 1974.

Bonanzinga, Sergio, La musica di tradizione orale in Lingue e culture in Sicilia a c. di Ruffino, Giovanni, Vol. 2, Palermo, Centro di studi filologici e linguistici siciliani, 2013.

${ }^{64}$ Bonanzinga, Sergio, op, cit., p. 194.

${ }^{65}$ Ivi, p. 644. [graduali intensificazioni del linguaggio popolare], traduzione nostra.

${ }^{66}$ Croce, Benedetto, Appunti di letteratura popolare da antiche opere letterarie, in Archivio per lo studio delle tradizioni popolari, rivista diretta da Pitré, Giuseppe e Salomone Marino, Salvatore Palermo-Torino, Clausen, n. 1-1894, p. 103. 
Cirese, Alberto Mario. Lo studio delle tradizioni popolari in Manuale di letteratura italiana. Storia per generi e problemi, volume quarto: Dali 'unità d 't talla alla fine del Novecento, a c. di F. Bnoschi e C. Di Girolamo. Torino, BoDati Boringhieri, 1996. Cocchiara Giuseppe, La lezione di Pitré, in Storia del folklore europeo, Torino, Boringhieri, 1979.

Cocchiara, Giuseppe. Pitrè, la Sicilia e il folklore, Messina-Firenze, G. D’Anna Editrice, 1951.

Croce, Benedetto, Appunti di letteratura popolare da antiche opere letterarie, in Archivio per lo studio delle tradizioni popolari, rivista diretta da Pitré, Giuseppe e Salomone Marino, Salvatore Palermo-Torino, Clausen, n. 1-1894.

Dardano Maurizio, Trifone Pietro. Grammatica italiana con nozioni di linguistica, Bologna, Zanichelli, 1995.

Debenedetti, Giacomo. Verga e il naturalismo, Milano, Aldo Garzanti Editore, 1978.

Fulci, Ludovico, La Lupa di Verga. Esercitazioni di lettura e spunti di approfondimento, Wrocław, Dolnośląskie Wydawnictwo Edukacyjne, 2005.

García Lorca, Federico, Bodas de sangre, Fuenlabrada (Madrid), Ediciones Cátedra, 2000.

García Lorca, Federico, Bodas de sangre; con cuadros cronológicos, intr., bibliografía, texto integro, notas y llamadas de atención, documentos y orientaciones para el estudio a c. di Manuel Cifo González, Madrid, Editorial Castalia, 1999.

García Lorca, Federico, conferencia dictada el 13 de diciembre de 1928: Las canciones de cuna españolas. En: Obras completas: México, Aguilar, 1991.

García Lorca, Federico, Od pierwszych pieśni do słów ostatnich : (wiersze, proza, listy, wypowiedzi), a c. di Szleyen, Zofia, Kraków, Wydawnictwo Literackie, 1987.

Giancristofaro, Lia. Il segno dei Vinti. Lettura antropologica dell'opera di Verga, https:// www.academia.edu/33137043/Il_segno_dei_Vinti._Lettura_antropologica_dellopera_di_Verga

González de Molina, Manuel. Algunas reflexiones sobre el mundo rural y los movimientos campesinos in Historia contemporánea española in Rivera, Antonio, Ortiz de Orruño, José María, Ugarte, Javier. Movimientos sociales en la España Contemporánea. Madrid, Abada Editores, 2008.

Lázaro Carreter, Fernando, Correa Calderón, Evaristo. Cómo se comenta un texto literario, Madrid, Ediciones Cátedra, 1975.

Luis Sanchez, Francisco, Pensamiento en la literatura española, Poznań, Wydawnictwo Naukowe UAM, 1992.

Muñiz Muñiz, María de las Nieves, 1999, Italia e Italie. Immagini tra Rivoluzione e Restaurazione, Roma, Bulzoni, pp. 161-181

Nicoll, Allardyce. Dzieje teatru, Warszawa, Państwowy Instytut Wydawniczy, 1959.

Pitrè, Giuseppe., Canti popolari siciliani, Bologna, Forni, 1980. 
Pitrè, Giuseppe. Usi e costumi, credenze e pregiudizi del popolo siciliano, Firenze, Barbera, 1944-1952.

Pitré, Giuseppe. Usi e costumi del popolo siciliano, Firenze, Universale Cappelli, 1961. Travalia, Caroline, Pazo, José, Juego, léxico y sintaxis en la lengua de García Lorca,

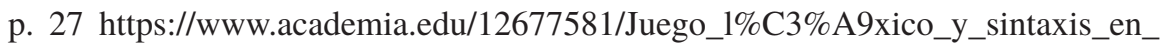
la_lengua_de_Garc\%C3\%ADa_Lorca

Raya, Gino, Carteggio Verga-Capuana, Roma, Edizioni dell'Ateneo, 1984

Senado Gomez, Julio. Castilla en escombros: las leyes, las tierras, el trigo y el hambre. Valladolid: Ámbito, 1993.

Scarfoglio, Edoardo. Il Libro Di Don Chisciotte - Primary Source Edition, Charleston, Nabu Press, 2014.

Sirvent del Otero. María Josefa, Actualidd del cuento infantil en España in Petrini, Enzo, Estudio crítico de la literatura juvenil, Madrid, Rialp, 1963.

Sansone, Marcello. Relazioni fra la letteratura italiana e le letterature dialettali, in Problemi e orientamenti critici di Lingua e di Letteratura italiana, IV (Letterature comparate), a c. di Momigliano, Arnoldo, Milano, Marzorati Editore, 1947.

77. Verga, Giovanni, La lupa in Tutto il teatro, Milano, Arnoldo Mondadori Editore, 1980.

Voce "compare" in Vocabolario Treccani http://www.treccani.it/vocabolario/compare/

Voce comare in Vocabolario Treccani http://www.treccani.it/vocabolario/comare/

Voce gnà in Vocabolario Treccani http://www.treccani.it/vocabolario/gna/

Zapullo Muscará, Sarah, Invito alla lettura di Verga, Milano, Mursia, 1977 (7ª ed., 1997).

Zappulla, Angelo, La Giustizia nella Letteratura e nello Spettacolo Siciliani tra '800 e '900. Da Verga a Sciascia, Catania, Istituto di Storia dello Spettacolo Siciliano, 1997. 
